\title{
Relationship of Metabolic Diseases with Physical Activity Depending on Age
}

Hyo Kyung Lim ${ }^{1}$, Jae Woong Sull ${ }^{2,3}$, Beom Seok Park ${ }^{2,3}$, Ji Young Mun ${ }^{2,3}$, Min Hwa Hong ${ }^{4}$, Yoori Lee ${ }^{4}$, Min Ji Hwang ${ }^{1}$, Mi Na Lee ${ }^{1}$, Ji Young Lee ${ }^{1}$, In Sik Kim ${ }^{1,4}$

${ }^{1}$ Department of Biomedical Laboratory Science, School of Medicine, Eulji University, Daejeon, Korea

${ }^{2}$ Department of Biomedical Laboratory Science, College of Health Science, Eulji University, Seongnam, Korea

${ }^{3}$ Department of Senior Healthcare, BK21 plus program, Graduate School, Eulji University, Seongnam, Korea

${ }^{4}$ Department of Senior Healthcare, BK21 plus program, Graduate School, Eulji University, Daejeon, Korea

\section{연령별 신체활동에 따른 대사성질환과의 관계}

\author{
임효경 ${ }^{1}$, 설재웅 ${ }^{2,3}$, 박범석 ${ }^{2,3}$, 문지영 ${ }^{2,3}$, 홍민화 $^{4}$, 이유리 ${ }^{4}$, 황민지 $^{1}$, 이미나 ${ }^{1}$, 이지영 ${ }^{1}$, 김인식 ${ }^{1,4}$ \\ ${ }^{1}$ 을지대학교 대전캠퍼스 임상병리학과, ${ }^{2}$ 을지대학교 성남캠퍼스 임상병리학과, ${ }^{3}$ 을지대학교 성남캠퍼스 BK21 플러스 프로그램 시니어 헬스케어학과, \\ 4을지대학교 대전캠퍼스 BK21 플러스 프로그램 시니어 헬스케어학과
}

\begin{abstract}
Metabolic disease is associated with abdominal obesity, high blood pressure, and dyslipidemia. Physical activity has beneficial effects on a variety of diseases. This study examined the relationship between metabolic diseases and physical activity according to age. Among a total of 7,295 subjects, the data from 382 individuals in the normal group and 1,525 persons in the metabolic disease group were analyzed. The data were analyzed statistically by one-way ANOVA, the Pearson's correlation coefficient, and multiple regression analysis. The levels of hemoglobin $(\mathrm{HB})$, hematocrit $(\mathrm{HCT})$, and creatinine (CR), were elevated when a high-intensity physical activity was performed, but they were reduced when a low-intensity physical activity was performed in the normal group aged 10 29 years and the metabolic disease group aged 50 69 years. In the normal group and metabolic disease group aged $30 \sim 49$ years, the level of high density lipoprotein cholesterol $(\mathrm{HDL}-\mathrm{C})$ was elevated when high-intensity physical activity was conducted, whereas it was reduced when low-intensity physical activity was performed. No difference in the level of $\mathrm{HDL}-\mathrm{C}$ depending on age and exercise intensity was observed in the normal group; the level of $\mathrm{HDL}-\mathrm{C}$ decreased with age and increased with exercise intensity in the metabolic disease group. Physical activity has different effects in metabolic disease depending on age.
\end{abstract}

Key words: Age, Metabolic disease, Physical activity

This is an Open Access article distributed under the terms of the Creative Commons Attribution Non-Commercial License (http://creativecommons.org/licenses/by-nc/4.0) which permits unrestricted non-commercial use, distribution, and reproduction in any medium, provided the original work is properly cited.

Copyright $\odot 2018$ The Korean Society for Clinical Laboratory Science. All rights reserved.
Corresponding author: In Sik Kim Department of Biomedical Laboratory Science, School of Medicine, Eulji University, Gyeryoung-ro 771-77, Jung-Gu, Daejeon 34824, Korea

Tel: 82-42-259-1753

Fax: 82-42-259-1759

E-mail: orientree@eulji.ac.kr

Received: April 26, 2018

Revised: May 24, 2018

Accepted: May 24, 2018

\section{INTRODUCTION}

Metabolic disease, which refers to a constellation of abdominal obesity, high blood pressure, dyslipidemia, and impaired fasting glucose (IFG) occurring in a single person, is known to increase the risk of developing cardiovascular disease and type 2 diabetes [1,2]. Although the pathogenesis of metabolic diseases has not yet been fully elucidated, it is known to be related to obesity, blood pressure, lipid levels, and body mass index [3, 4]. The prevalence of metabolic syndrome was $23 \%$ in a survey of 8,814 persons in urban areas of the United States according 
to the NCEP-ATPIII criteria, while it was $16.7 \%$ in a survey among the Hong Kong population [5]. Additionally, the 4th Korea National Health and Nutrition Examination Survey found that the prevalence of metabolic syndrome in adults in Korea is $32.4 \%$, which is very high compared with the prevalence rates of other countries throughout the world [6].

Efforts to manage chronic diseases have led to attempts to change behaviors related to personal lifestyle, and the most important health-promoting behavior has been found to be physical activity [7]. Moreover, previous studies have shown that physical activity reduces the risk of metabolic diseases [8-10]. Regular physical activity has beneficial effects that improve cardiopulmonary functions and delays metabolic diseases associated with aging and chronic degenerative diseases [11-14]. There are many types of regular physical activity that adults can participate in. Regular walking is one of the easiest physical activities that can be performed safely anywhere at any time. In addition, several previous studies have reported that regular walking for 30 minutes or more a day reduces the risk of developing metabolic diseases [15, 16]. However, the effects of high-intensity physical activity including regular walking vary greatly. Participation in high-intensity physical activity is known to have more positive effects on physical strength (cardiovascular fitness), body composition and biochemical changes than participation in lowintensity or moderate-intensity physical activity [17, 18]. In particular, if the amount of energy consumed during physical activity is constant, participation in high intensity physical activity can lead to more effective improvement in risk factors associated with cardiovascular diseases than participation in low intensity or moderate intensity physical activity [19]. Rennie et al found that the group participating in high intensity physical activity showed a greater decrease in the risk of developing metabolic disease than the group not participating in physical activity [20]. Moreover, reductions in physical activity have been reported to be closely related to the incidence of metabolic diseases and many adult diseases [21, 22]. Nevertheless, there is a lack of research regarding the age-related association between metabolic diseases and physical activity. Therefore, this study was conducted to investigate the parameters of metabolic diseases in groups participating in high-intensity, moderate-intensity, and low-intensity physical activity according to age.

\section{MATERIALS AND METHODS}

\section{Ethical approval}

The study was reviewed and approved by the Ethics Committee of the Korea Centers for Disease Control and Prevention (Approval Number 2013-12EXP-03-5C).

\section{Subjects}

This study was conducted using the second-year survey data of the 6th Korea National Health and Nutrition Examination Survey (KNHANES VI-2) conducted from January to December in 2014. The data used in this study were provided according to the procedures presented in the KNHANES homepage (https://knhanes.cdc.go.kr) [23]. Out of a total of 9,701 respondents aged 19 or older, 2,506 were excluded. Reasons for exclusion included an absence of data regarding physical activity or parameters known to be associated with metabolic diseases, such as blood pressure (BP), waist circumference (WC), body mass index (BMI) and the levels of red blood cells (RBC), hemoglobin (HB), hematocrit (HCT), white blood cells (WBC), platelets (PLT), fasting blood sugars (FBS), triglycerides (TG), high density lipoprotein cholesterol (HDL-C), aspartate aminotransferase (AST), alanine aminotransaminase (ALT), total cholesterol (TC), blood urea nitrogen (BUN), and creatinine (CR). Thus, the data from 7,295 subjects were analyzed. Among these, 382 persons in the normal group and 1,525 persons in the metabolic disease group were analyzed to investigate the relationship between metabolic diseases and physical activity of lowintensity, moderate-intensity and high-intensity according to age using physical, hematological and biochemical parameters.

\section{Investigation}

The criteria for the diagnosis of metabolic diseases were 
defined using the guidelines presented in the National Cholesterol Education Program Adults Treatment Panel III; NCEP ATP III [24]. Briefly, the criteria for diagnosis of metabolic diseases were fasting blood glucose $(\geq 110$ $\mathrm{mg} / \mathrm{dL}$ ), Asian waist circumference (male $\geq 90 \mathrm{~cm}$, female $>80 \mathrm{~cm}$ ), blood pressure (systolic/diastolic blood pressure $\geq 130 / 85 \mathrm{mmHg}$ ), triglycerides ( $\geq 150 \mathrm{mg} / \mathrm{dL}$ ), and high density lipoprotein cholesterol (male $<40 \mathrm{mg} / \mathrm{dL}$, female $<50 \mathrm{mg} / \mathrm{dL}$ ) When three or more of the criteria were met, the patient was diagnosed with metabolic disease.

Physical activity was measured using a Korean-version short-form self-report measure of the International Physical Activity Questionnaire developed for the purpose of comprehensive and objective assessment of daily physical activity in everyday life as well as health-related physical activity [25]. The measurement tool was designed to respond to the vigorous physical activity, moderate physical activity, and walking time of 10 minutes or more during the 7 days before the questionnaire survey. Vigorous physical activity was considered activity that makes you breathe much more heavily than usual, and included carrying heavy objects, running, aerobic exercises, climbing, and cycling at a fast speed. Moderate physical activity was defined as activity that led to slightly heavier breath than usual, such as carrying light items, biking at a normal speed, dancing, etc. Walking included walking during recreational activities, sports, exercise, and leisure time, as well as walking at work, home, and while using transportation. The amount of physical activity was converted into the continuous index and categorical index according to the Guidelines for Data Processing and Analysis of the International Physical Activity Questionnaire. The continuous index is for determining the Metabolic Equivalent Task (MET) to compare levels of energy consumption by multiplying the MET level by each activity time. The MET level of each physical activity is 8 for vigorous physical activity, 4 for moderate physical activity, and 3.3 for walking. The categorical index indicates the division of subjects into three levels according to the following criteria. The inactive group, Group 1 (lowintensity), was the group of people who perform the lowest degree of physical activity. This group includes those who do not belong to Group 2 (moderate-intensity) or Group 3 (high- intensity) or do not perform physical activity. Group 2, which was the minimum physical activity group, included people that satisfy any one of the following three criteria: vigorous physical activity for at least 20 minutes per day for at least three days a week; moderate physical activity or walking for at least 30 minutes per day for at least five days a week; physical activity of at least $600 \mathrm{MET}$-min/week through walking at least 5 days a week or through any combination of moderate or vigorous physical activity. Finally, Group 3, which was the health promoting activity group, included people that satisfied one of the following two criteria: consume at least 1,500 MET-min/week through vigorous activity at least three days per week, or consume at least 3,000 MET-min/week through walking 7 days a week or through any combination of moderate or vigorous physical activity [26]. The amount of physical activity was calculated by measuring the time of vigorous physical activity, moderate physical activity, walking, and sedentary activity in the past 7 days, and then converting them to MET (min/week) values to derive the continuous index and categorical index. The total physical activity score of the continuous score was calculated as the sum of the MET values of walking, moderate-activity, and vigorous activity, while physical activity of less than 10 minutes was considered to be equivalent to no physical activity.

\section{Statistical analysis}

The data collected in this study were statistically analyzed using the SPSS version 20 program. Descriptive statistics were used to determine the general characteristics of the subjects. ANOVA (analysis of variance) was performed to examine the differences between the normal and metabolic disease groups according to age and the differences depending on physical activity according to age. Correlations between age and metabolic parameters in the normal and metabolic disease groups were confirmed using Pearson's correlation coefficient. An independent sample t-test was conducted to examine the 
differences between normal and metabolic disease groups. Multiple regression analysis was conducted to investigate the effects of age and physical activity intensity on parameters of metabolic diseases in the normal and metabolic disease groups.

\section{RESULTS}

\section{Correlation between age and metabolic parameters in the normal and metabolic disease groups}

For physical, hematological, and biochemical characteristics according to age in the normal and metabolic disease groups, there were statistically significant differences in

Table 1. General characteristics of the normal and metabolic disease groups according to age

\begin{tabular}{|c|c|c|c|c|c|c|c|}
\hline \multicolumn{3}{|c|}{ Age Characteristic } & \multirow{2}{*}{$\frac{10 \sim 29}{217}$} & \multirow{2}{*}{$\frac{30 \sim 49}{144}$} & \multirow{2}{*}{$\frac{50 \sim 69}{21}$} & \multirow[t]{2}{*}{$\mathrm{F}$} & \multirow[t]{2}{*}{$P$} \\
\hline Physical & Normal & $\mathrm{N}$ & & & & & \\
\hline & & WC & $70.37 \pm 7.23$ & $72.69 \pm 7.01$ & $79.63 \pm 9.22$ & $17.478^{\star \star}$ & 0.000 \\
\hline & & BMI & $20.69 \pm 2.52$ & $21.26 \pm 2.57$ & $23.14 \pm 2.68$ & $9.696^{\star \star}$ & 0.000 \\
\hline & & SBP & $103.18 \pm 6.91$ & $100.76 \pm 6.88$ & $103.95 \pm 7.97$ & $5.819^{\star \star}$ & 0.003 \\
\hline & & DBP & $65.57 \pm 6.18$ & $67.49 \pm 5.44$ & $69.43 \pm 6.03$ & $7.299^{\star \star}$ & 0.001 \\
\hline & Metabolic & N & 97 & 486 & 942 & & \\
\hline & & WC & $76.43 \pm 13.21$ & $85.14 \pm 9.53$ & $85.03 \pm 8.78$ & $38.627^{\star \star}$ & 0.000 \\
\hline & & BMI & $23.17 \pm 5.02$ & $25.34 \pm 3.52$ & $24.87 \pm 3.11$ & $16.764^{\star \star}$ & 0.000 \\
\hline & & SBP & $114.29 \pm 12.24$ & $118.29 \pm 15.43$ & $125.71 \pm 16.90$ & $47.338^{\star \star}$ & 0.000 \\
\hline & & DBP & $71.69 \pm 11.29$ & $80.23 \pm 11.40$ & $78.31 \pm 10.33$ & $25.890^{\star \star}$ & 0.000 \\
\hline \multirow[t]{12}{*}{ Hematological } & Normal & $\mathrm{N}$ & 217 & 144 & 21 & & \\
\hline & & WBC & $6.25 \pm 1.19$ & $5.75 \pm 1.16$ & $5.96 \pm 1.10$ & $7.826^{\star \star}$ & 0.000 \\
\hline & & $\mathrm{RBC}$ & $4.62 \pm 0.39$ & $4.44 \pm 0.34$ & $4.37 \pm 0.33$ & $11.329^{\star \star}$ & 0.000 \\
\hline & & $\mathrm{HB}$ & $13.99 \pm 1.27$ & $13.57 \pm 1.12$ & $14.01 \pm 1.11$ & $5.443^{\star \star}$ & 0.005 \\
\hline & & $\mathrm{HCT}$ & $41.46 \pm 3.22$ & $40.38 \pm 2.79$ & $41.25 \pm 2.95$ & $5.574^{\star \star}$ & 0.004 \\
\hline & & PLT & $263.08 \pm 44.39$ & $258.62 \pm 50.81$ & $255.33 \pm 42.77$ & 0.555 & 0.575 \\
\hline & Metabolic & $\mathrm{N}$ & 97 & 486 & 942 & & \\
\hline & & WBC & $6.93 \pm 1.51$ & $6.87 \pm 1.76$ & $6.38 \pm 1.78$ & $14.732^{\star \star}$ & 0.000 \\
\hline & & RBC & $4.97 \pm 0.43$ & $4.82 \pm 0.43$ & $4.60 \pm 0.41$ & $68.580^{\star *}$ & 0.000 \\
\hline & & $\mathrm{HB}$ & $14.68 \pm 1.48$ & $14.80 \pm 1.61$ & $14.36 \pm 1.42$ & $14.375^{\star \star}$ & 0.000 \\
\hline & & $\mathrm{HCT}$ & $43.25 \pm 3.70$ & $43.60 \pm 4.11$ & $42.40 \pm 3.71$ & $16.079^{\star \star}$ & 0.000 \\
\hline & & PLT & $288.01 \pm 56.95$ & $267.07 \pm 55.32$ & $249.5 \pm 57.24$ & $30.318^{\star \star}$ & 0.000 \\
\hline \multirow{18}{*}{ Biochemical } & Normal & $N$ & 217 & 144 & 21 & & \\
\hline & & FBS & $86.87 \pm 5.42$ & $87.10 \pm 6.83$ & $88.71 \pm 5.77$ & 0.906 & 0.405 \\
\hline & & $\mathrm{TC}$ & $157.91 \pm 21.73$ & $166.75 \pm 20.08$ & $169.05 \pm 19.12$ & $8.985^{\star \star}$ & 0.000 \\
\hline & & $\mathrm{TG}$ & $64.26 \pm 22.94$ & $66.92 \pm 23.23$ & $74.4 \pm 19.48$ & 2.156 & 0.117 \\
\hline & & $\mathrm{HDL}$ & $56.79 \pm 9.36$ & $57.69 \pm 10.76$ & $53.18 \pm 10.17$ & 1.933 & 0.146 \\
\hline & & BUN & $11.42 \pm 2.92$ & $12.31 \pm 3.06$ & $13.95 \pm 3.22$ & $9.114^{\star \star}$ & 0.000 \\
\hline & & $C R$ & $0.73 \pm 0.12$ & $0.73 \pm 0.12$ & $0.72 \pm 0.13$ & 0.162 & 0.851 \\
\hline & & AST & $16.71 \pm 3.88$ & $16.84 \pm 3.26$ & $21.14 \pm 3.69$ & $14.347^{\star \star}$ & 0.000 \\
\hline & & ALT & $12.42 \pm 5.51$ & $12.74 \pm 4.53$ & $17.14 \pm 6.31$ & $7.884^{\star \star}$ & 0.000 \\
\hline & Metabolic & $N$ & 97 & 486 & 942 & & \\
\hline & & FBS & $106.80 \pm 26.06$ & $115.10 \pm 34.00$ & $117.12 \pm 28.96$ & $5.225^{\star \star}$ & 0.005 \\
\hline & & $\mathrm{TC}$ & $174.58 \pm 44.37$ & $207.25 \pm 39.76$ & $200.33 \pm 40.35$ & $26.627^{\star \star}$ & 0.000 \\
\hline & & $\mathrm{TG}$ & $109.26 \pm 71.89$ & $196.15 \pm 170.24$ & $167.91 \pm 112.10$ & $19.571^{\star \star}$ & 0.000 \\
\hline & & $\mathrm{HDL}$ & $53.67 \pm 11.27$ & $49.62 \pm 12.02$ & $49.28 \pm 11.74$ & $5.833^{\star \star}$ & 0.003 \\
\hline & & BUN & $12.77 \pm 3.81$ & $13.87 \pm 4.08$ & $15.77 \pm 4.51$ & $43.881^{\star \star}$ & 0.000 \\
\hline & & $C R$ & $0.77 \pm 0.18$ & $0.84 \pm 0.17$ & $0.85 \pm 0.29$ & $4.454^{*}$ & 0.012 \\
\hline & & AST & $20.43 \pm 11.73$ & $24.02 \pm 15.76$ & $25.33 \pm 15.66$ & $4.918^{\star \star}$ & 0.007 \\
\hline & & $\mathrm{ALT}$ & $21.89 \pm 22.04$ & $28.38 \pm 22.83$ & $25.42 \pm 18.59$ & $5.712^{\star \star}$ & 0.003 \\
\hline
\end{tabular}

${ }^{\star} p<0.05,{ }^{* *} p<0.01$.

Abbreviations: N, number; WC, waist circumference; BMI, body mass index; SBP, systolic blood pressure; DBP, diastolic blood pressure; WBC, white blood cell, RBC, red blood cell; HB, hemoglobin; HCT, hematocrit; PLT, platelet; FBS, fasting blood sugars; TC, total cholesterol; TG, triglycerides; HDL, high density lipoprotein; BUN, blood urea nitrogen; CR, creatinine; AST, aspartate aminotransferase; ALT, alanine aminotransaminase. 
the levels of various factors (Table 1). In the normal group, SBP $(P<0.01)$, WBC $(P<0.01), \mathrm{RBC}(P<0.01), \mathrm{HB}(P<$ $0.05)$, and $\mathrm{HCT}(P<0.01)$ were found to be negatively correlated with age (Table 2). Additionally, WC $(P<0.01)$, BMI $(P<0.01)$, DBP $(P<0.01)$, TC $(P<0.01)$, BUN $(P<$ $0.01)$, AST $(P<0.01)$ and ALT $(P<0.01)$ were found to be positively correlated with age. In the metabolic disease group, $\mathrm{WBC}(P<0.01), \mathrm{RBC}(P<0.01), \mathrm{HB}(P<0.01), \mathrm{HCT}$ $(P<0.01)$, PLT $(P<0.01)$, and HDL-C $(P<0.01)$ were found to be negatively correlated with age. WC $(P<0.01)$, SBP $(P<0.01)$, FBS $(P<0.01), \mathrm{BUN}(P<0.01), \mathrm{CR}(P<0.01)$, and AST $(P<0.05)$ were found to have a positive correlation with age.

\section{The relationship between physical activity and parameters of metabolic diseases by age}

The relationship of metabolic diseases with physical activity according to age was examined. Among people aged 10 29 years in the normal group, the levels of significant variables of metabolic diseases (RBC, HB, HCT, $\mathrm{CR}$, and BUN) increased when high-intensity physical activity was performed and decreased when low-intensity physical activity was performed. No significant variables were identified in the metabolic disease group. Among

Table 2. Correlation between age and metabolic parameters in the normal and metabolic disease groups

\begin{tabular}{|c|c|c|c|c|}
\hline \multirow{2}{*}{ Parameters } & \multicolumn{2}{|c|}{ Normal $(\mathrm{N}=382)$} & \multicolumn{2}{|c|}{ Metabolic $(\mathrm{N}=1,525)$} \\
\hline & r & $P$ & r & $P$ \\
\hline WC & $0.279^{\star \star}$ & 0.000 & $0.159^{\star *}$ & 0.000 \\
\hline BMI & $0.217^{\star \star}$ & 0.000 & 0.043 & 0.091 \\
\hline SBP & $-0.142^{\star *}$ & 0.006 & $0.237^{\star \star}$ & 0.000 \\
\hline DBP & $0.204^{* \star}$ & 0.000 & 0.005 & 0.831 \\
\hline WBC & $-0.195^{\star \star}$ & 0.000 & $-0.115^{\star \star}$ & 0.000 \\
\hline $\mathrm{RBC}$ & $-0.270^{\star \star}$ & 0.000 & $-0.305^{\star \star}$ & 0.000 \\
\hline $\mathrm{HB}$ & $-0.117^{\star}$ & 0.022 & $-0.125^{\star \star}$ & 0.000 \\
\hline $\mathrm{HCT}$ & $-0.135^{\star \star}$ & 0.008 & $-0.132^{\star \star}$ & 0.000 \\
\hline PLT & -0.083 & 0.105 & $-0.198^{\star \star}$ & 0.000 \\
\hline FBS & 0.002 & 0.971 & $0.096^{\star \star}$ & 0.000 \\
\hline $\mathrm{TC}$ & $0.250^{\star \star}$ & 0.000 & 0.034 & 0.186 \\
\hline TG & 0.060 & 0.239 & 0.011 & 0.681 \\
\hline $\mathrm{HDL}-\mathrm{C}$ & 0.039 & 0.453 & $-0.097^{\star \star}$ & 0.000 \\
\hline BUN & $0.235^{\star \star}$ & 0.000 & $0.250^{\star \star}$ & 0.000 \\
\hline$C R$ & 0.032 & 0.527 & $0.088^{\star *}$ & 0.001 \\
\hline AST & $0.139 * \star$ & 0.007 & $0.064^{*}$ & 0.012 \\
\hline ALT & $0.133^{\star \star}$ & 0.009 & -0.037 & 0.145 \\
\hline
\end{tabular}

${ }^{*} P<0.05,{ }^{*} p<0.01$. those aged 30 49 years in the normal group and metabolic disease groups, HDL-C, which is a significant variable for metabolic diseases, increased when high-intensity physical activity was performed and decreased when low-intensity physical activity was performed. No significant variables of metabolic diseases were identified in 50 69 year olds of the normal group. In the metabolic disease group, significant variables of metabolic diseases (HB, HCT, CR) were elevated when high-intensity physical activity was performed and reduced when low-intensity physical activity was performed. The relationships between parameters of metabolic diseases and physical activity according to age in the normal group and metabolic disease group are summarized in Tables $3 \sim 6$.

\section{Effects of age and physical activity intensity on $\mathrm{HDL}-\mathrm{C}$ in the normal and metabolic disease groups}

Table 7 shows the effects of age and physical activity intensity on HDL-C in the normal and metabolic disease groups. The results of the analysis showed that there was no statistically significant difference in HDL-C depending on age and exercise intensity in the normal group, and regression equation was not significant. However, in the metabolic disease group, the HDL-C level was found to decrease with age $(\beta=-0.09)$ and increase with physical activity intensity ( $\beta=0.07$ ). In other words, in the metabolic disease group, as more physical activities were performed the HDL-C level was elevated, the regression equation became significant and the explanatory power was $1.3 \%$.

Table 3. The relationship between physical activity and parameters of metabolic diseases by age

\begin{tabular}{cccc}
\hline Age & Group & $\begin{array}{c}\text { Metabolic disease } \\
\text { parameters }\end{array}$ & $\begin{array}{c}\text { Physical activity } \\
\text { results }\end{array}$ \\
\hline $10 \sim 29$ yrs & Normal & RBC, HB, HCT, & High intensity $\uparrow$, \\
& Metabolic & - & Low intensity $\downarrow$ \\
$30 \sim 49$ yrs & Normal & HDL-C & - \\
& Metabolic & HDL-C & $\begin{array}{c}\text { High intensity } \uparrow, \\
\text { Low intensity } \downarrow \\
\text { High intensity } \uparrow, \\
\text { Low intensity } \downarrow\end{array}$ \\
$50 \sim 69$ yrs & Normal & - & - \\
& Metabolic & HB, HCT, CR & High intensity $\uparrow$, \\
& & & Low intensity $\downarrow$ \\
\hline
\end{tabular}


Table 4. Physical, hematological, and biochemical characteristics according to physical activity by age (10 29 years)

\begin{tabular}{|c|c|c|c|c|c|c|c|}
\hline \multicolumn{3}{|c|}{ Characteristic } & \multirow{2}{*}{$\begin{array}{l}\text { Low } \\
122\end{array}$} & \multirow{2}{*}{$\begin{array}{c}\text { Moderate } \\
78\end{array}$} & \multirow{2}{*}{$\begin{array}{c}\text { High } \\
17\end{array}$} & \multirow[t]{2}{*}{$\mathrm{F}$} & \multirow[t]{2}{*}{$P$} \\
\hline Physical & Normal & $\mathrm{N}$ & & & & & \\
\hline & & Age & $21.46 \pm 5.21$ & $19.45 \pm 4.80$ & $19.53 \pm 4.72$ & $4.205^{\star}$ & 0.016 \\
\hline & & WC & $69.77 \pm 7.18$ & $70.8 \pm 7.34$ & $72.66 \pm 6.83$ & 1.428 & 0.242 \\
\hline & & BMI & $20.49 \pm 2.45$ & $20.85 \pm 2.68$ & $21.39 \pm 2.19$ & 1.189 & 0.307 \\
\hline & & SBP & $102.79 \pm 7.03$ & $103.79 \pm 7.06$ & $103.12 \pm 5.28$ & 0.505 & 0.604 \\
\hline & & DBP & $65.77 \pm 6.59$ & $65.50 \pm 5.68$ & $64.41 \pm 5.49$ & 0.365 & 0.694 \\
\hline & Metabolic & $N$ & 57 & 28 & 12 & & \\
\hline & & Age & $17.63 \pm 5.97$ & $19.07 \pm 5.40$ & $18.25 \pm 5.10$ & 0.600 & 0.551 \\
\hline & & WC & $75.33 \pm 12.37$ & $79.5 \pm 13.82$ & $74.5 \pm 15.50$ & 1.085 & 0.342 \\
\hline & & BMI & $22.72 \pm 4.90$ & $24.35 \pm 5.13$ & $22.59 \pm 5.26$ & 1.095 & 0.339 \\
\hline & & SBP & $113.47 \pm 12.20$ & $116.14 \pm 13.57$ & $113.83 \pm 9.23$ & 0.451 & 0.639 \\
\hline & & DBP & $70.77 \pm 11.82$ & $74.18 \pm 11.44$ & $70.25 \pm 7.50$ & 0.967 & 0.384 \\
\hline \multirow[t]{12}{*}{ Hematological } & Normal & $\mathrm{N}$ & 122 & 78 & 17 & & \\
\hline & & WBC & $6.13 \pm 1.14$ & $6.39 \pm 1.27$ & $6.45 \pm 1.10$ & 1.362 & 0.258 \\
\hline & & $\mathrm{RBC}$ & $4.56 \pm 0.36$ & $4.67 \pm 0.41$ & $4.75 \pm 0.46$ & $3.149^{\star}$ & 0.045 \\
\hline & & $\mathrm{HB}$ & $13.82 \pm 1.20$ & $14.10 \pm 1.30$ & $14.66 \pm 1.42$ & $3.861^{\star}$ & 0.023 \\
\hline & & $\mathrm{HCT}$ & $40.98 \pm 3.01$ & $41.91 \pm 3.32$ & $42.88 \pm 3.67$ & $3.850^{\star}$ & 0.023 \\
\hline & & PLT & $262.56 \pm 41.87$ & $264.37 \pm 48.27$ & $260.94 \pm 45.97$ & 0.061 & 0.941 \\
\hline & Metabolic & $N$ & 57 & 28 & 12 & & \\
\hline & & WBC & $7.08 \pm 1.54$ & $6.72 \pm 1.45$ & $6.76 \pm 1.58$ & 0.619 & 0.541 \\
\hline & & $\mathrm{RBC}$ & $4.99 \pm 0.41$ & $4.89 \pm 0.46$ & $5.11 \pm 0.44$ & 1.150 & 0.321 \\
\hline & & $\mathrm{HB}$ & $14.64 \pm 1.47$ & $14.6 \pm 1.63$ & $15.01 \pm 1.22$ & 0.343 & 0.711 \\
\hline & & $\mathrm{HCT}$ & $43.1 \pm 3.71$ & $43.1 \pm 3.98$ & $44.33 \pm 2.99$ & 0.579 & 0.562 \\
\hline & & PLT & $287.28 \pm 61.86$ & $285.39 \pm 50.52$ & $297.58 \pm 49.35$ & 0.200 & 0.819 \\
\hline \multirow[t]{18}{*}{ Biochemical } & Normal & $\mathrm{N}$ & 122 & 78 & 17 & & \\
\hline & & FBS & $86.52 \pm 5.54$ & $87.15 \pm 5.47$ & $88.12 \pm 4.21$ & 0.815 & 0.444 \\
\hline & & $\mathrm{TC}$ & $158.92 \pm 21.00$ & $156.27 \pm 23.21$ & $158.18 \pm 20.63$ & 0.353 & 0.703 \\
\hline & & $\mathrm{TG}$ & $63.52 \pm 22.34$ & $65.17 \pm 24.17$ & $65.41 \pm 22.48$ & 0.144 & 0.866 \\
\hline & & $\mathrm{HDL}$ & $56.83 \pm 9.30$ & $56.68 \pm 9.92$ & $57.04 \pm 7.40$ & 0.012 & 0.988 \\
\hline & & BUN & $11.25 \pm 2.73$ & $11.15 \pm 3.01$ & $13.88 \pm 2.96$ & $6.927^{\star \star}$ & 0.001 \\
\hline & & $C R$ & $0.72 \pm 0.12$ & $0.74 \pm 0.13$ & $0.82 \pm 0.14$ & $4.752^{\star \star}$ & 0.010 \\
\hline & & AST & $16.73 \pm 4.01$ & $16.79 \pm 3.86$ & $16.18 \pm 3.13$ & 0.179 & 0.836 \\
\hline & & ALT & $12.61 \pm 6.12$ & $11.97 \pm 4.64$ & $13.06 \pm 4.70$ & 0.443 & 0.643 \\
\hline & Metabolic & $\mathrm{N}$ & 57 & 28 & 12 & & \\
\hline & & FBS & $108.07 \pm 30.59$ & $105.46 \pm 21.34$ & $103.92 \pm 4.96$ & 0.175 & 0.840 \\
\hline & & $\mathrm{TC}$ & $169.93 \pm 36.85$ & $189.43 \pm 53.66$ & $162.00 \pm 48.54$ & 2.434 & 0.093 \\
\hline & & $\mathrm{TG}$ & $113.96 \pm 79.51$ & $105.36 \pm 62.34$ & $96.00 \pm 55.10$ & 0.363 & 0.697 \\
\hline & & $\mathrm{HDL}$ & $53.47 \pm 11.52$ & $52.96 \pm 10.02$ & $56.15 \pm 13.30$ & 0.348 & 0.707 \\
\hline & & BUN & $13.09 \pm 4.26$ & $12.68 \pm 3.41$ & $11.50 \pm 1.88$ & 0.870 & 0.422 \\
\hline & & $C R$ & $0.74 \pm 0.18$ & $0.79 \pm 0.20$ & $0.83 \pm 0.13$ & 1.613 & 0.205 \\
\hline & & AST & $20.05 \pm 8.73$ & $21.46 \pm 17.88$ & $19.83 \pm 4.86$ & 0.151 & 0.860 \\
\hline & & ALT & $20.40 \pm 17.84$ & $25.82 \pm 31.94$ & $19.75 \pm 7.71$ & 0.627 & 0.537 \\
\hline
\end{tabular}

${ }^{*} p<0.05,{ }^{*} p<0.01$.

\section{DISCUSSION}

This study was conducted to investigate the relationship between parameters of metabolic diseases and physical activity according to age in normal adults and those with metabolic disease, as well as to provide basic data for prevention of metabolic diseases depending on physical activity according to age. Overall, about $45 \%$ of adults engage in low-intensity physical activity, while 19.7\% perform moderate-intensity physical activity, and 35.4\% engage in high-intensity physical activity. Participation in high-intensity physical activity has been reported to have a positive effect on various parameters of metabolic diseases [27]. In a study of 612 adult males with no metabolic disease by Laaksonen et al [28], which was controlled for age and BMI, the incidence rate of metabolic 
150 Hyo Kyung Lim, et al. Relationship of Metabolic Diseases with Physical Activity Depending on Age

Table 5. Physical, hematological, and biochemical characteristics according to physical activity by age (30 49 years)

\begin{tabular}{|c|c|c|c|c|c|c|c|}
\hline \multicolumn{3}{|c|}{ Characteristic } & \multirow{2}{*}{$\frac{\text { Low }}{88}$} & \multirow{2}{*}{$\frac{\text { Moderate }}{49}$} & \multirow{2}{*}{$\begin{array}{c}\text { High } \\
7\end{array}$} & \multirow[t]{2}{*}{$\mathrm{F}$} & \multirow[t]{2}{*}{$P$} \\
\hline Physical & Normal & N & & & & & \\
\hline & & Age & $37.55 \pm 5.35$ & $37.08 \pm 4.87$ & $38.57 \pm 5.53$ & 0.300 & 0.741 \\
\hline & & WC & $72.62 \pm 7.00$ & $72.77 \pm 7.28$ & $73.06 \pm 6.06$ & 0.018 & 0.983 \\
\hline & & BMI & $21.06 \pm 2.32$ & $21.56 \pm 3.04$ & $21.66 \pm 2.17$ & 0.660 & 0.519 \\
\hline & & SBP & $99.99 \pm 7.07$ & $102.04 \pm 6.49$ & $101.57 \pm 6.48$ & 1.462 & 0.235 \\
\hline & & DBP & $66.69 \pm 5.71$ & $68.76 \pm 4.92$ & $68.57 \pm 3.95$ & 2.455 & 0.090 \\
\hline & Metabolic & $\mathrm{N}$ & 351 & 111 & 24 & & \\
\hline & & Age & $41.14 \pm 5.57$ & $40.68 \pm 5.11$ & $40.21 \pm 5.33$ & 0.551 & 0.577 \\
\hline & & WC & $85.59 \pm 9.73$ & $84.19 \pm 9.13$ & $82.86 \pm 7.78$ & 1.637 & 0.196 \\
\hline & & BMI & $25.38 \pm 3.55$ & $25.32 \pm 3.56$ & $24.97 \pm 2.94$ & 0.156 & 0.855 \\
\hline & & SBP & $118.09 \pm 14.92$ & $118.61 \pm 16.15$ & $119.71 \pm 19.62$ & 0.154 & 0.857 \\
\hline & & DBP & $80.03 \pm 10.75$ & $80.73 \pm 13.03$ & $80.67 \pm 13.01$ & 0.175 & 0.840 \\
\hline \multirow[t]{12}{*}{ Hematological } & Normal & $N$ & 88 & 49 & 7 & & \\
\hline & & WBC & $5.89 \pm 1.03$ & $5.48 \pm 1.26$ & $5.92 \pm 1.79$ & 2.000 & 0.139 \\
\hline & & $\mathrm{RBC}$ & $4.48 \pm 0.36$ & $4.42 \pm 0.31$ & $4.18 \pm 0.16$ & 2.582 & 0.079 \\
\hline & & $\mathrm{HB}$ & $13.72 \pm 1.15$ & $13.40 \pm 1.11$ & $12.84 \pm 0.26$ & 2.846 & 0.061 \\
\hline & & HCT & $40.70 \pm 2.81$ & $40.05 \pm 2.84$ & $38.53 \pm 0.79$ & 2.521 & 0.084 \\
\hline & & PLT & $259.61 \pm 48.72$ & $259.92 \pm 54.74$ & $237.00 \pm 50.51$ & 0.664 & 0.517 \\
\hline & Metabolic & $\mathrm{N}$ & 351 & 111 & 24 & & \\
\hline & & WBC & $6.98 \pm 1.72$ & $6.62 \pm 1.94$ & $6.54 \pm 1.33$ & 2.207 & 0.111 \\
\hline & & $\mathrm{RBC}$ & $4.84 \pm 0.43$ & $4.77 \pm 0.42$ & $4.85 \pm 0.45$ & 1.067 & 0.345 \\
\hline & & $\mathrm{HB}$ & $14.87 \pm 1.58$ & $14.56 \pm 1.67$ & $14.95 \pm 1.62$ & 1.642 & 0.195 \\
\hline & & HCT & $43.77 \pm 4.06$ & $42.98 \pm 4.21$ & $44.01 \pm 4.21$ & 1.684 & 0.187 \\
\hline & & PLT & $265.88 \pm 56.58$ & $271.03 \pm 51.14$ & $266.29 \pm 56.58$ & 0.364 & 0.695 \\
\hline \multirow{18}{*}{ Biochemical } & Normal & N & 88 & 49 & 7 & & \\
\hline & & FBS & $87.01 \pm 6.30$ & $86.78 \pm 7.80$ & $90.43 \pm 6.00$ & 0.893 & 0.412 \\
\hline & & $\mathrm{TC}$ & $166.80 \pm 20.30$ & $166.49 \pm 18.81$ & $168.00 \pm 28.32$ & 0.018 & 0.983 \\
\hline & & TG & $69.08 \pm 24.68$ & $64.86 \pm 20.30$ & $54.29 \pm 21.20$ & 1.622 & 0.201 \\
\hline & & $\mathrm{HDL}$ & $56.78 \pm 10.167$ & $57.03 \pm 9.010$ & $73.86 \pm 17.00$ & $9.276^{\star \star}$ & 0.000 \\
\hline & & BUN & $12.17 \pm 3.28$ & $12.47 \pm 2.72$ & $13.00 \pm 2.52$ & 0.333 & 0.717 \\
\hline & & $C R$ & $0.73 \pm 0.12$ & $0.73 \pm 0.11$ & $0.64 \pm 0.12$ & 1.899 & 0.154 \\
\hline & & AST & $16.25 \pm 2.84$ & $17.80 \pm 3.78$ & $17.57 \pm 3.10$ & $3.864^{\star}$ & 0.023 \\
\hline & & ALT & $12.39 \pm 4.45$ & $13.57 \pm 4.80$ & $11.43 \pm 2.64$ & 1.398 & 0.251 \\
\hline & Metabolic & $N$ & 351 & 111 & 24 & & \\
\hline & & FBS & $114.23 \pm 29.08$ & $118.13 \pm 43.32$ & $113.92 \pm 50.12$ & 0.563 & 0.570 \\
\hline & & TC & $206.00 \pm 40.41$ & $208.77 \pm 37.67$ & $218.50 \pm 39.01$ & 1.216 & 0.297 \\
\hline & & TG & $199.24 \pm 162.29$ & $184.50 \pm 167.88$ & $204.29 \pm 273.20$ & 0.342 & 0.711 \\
\hline & & $\mathrm{HDL}$ & $48.90 \pm 11.12$ & $50.89 \pm 14.31$ & $54.28 \pm 11.91$ & $2.976^{\star}$ & 0.050 \\
\hline & & BUN & $13.84 \pm 4.09$ & $14.05 \pm 4.11$ & $13.58 \pm 3.82$ & 0.169 & 0.844 \\
\hline & & $C R$ & $0.84 \pm 0.17$ & $0.86 \pm 0.19$ & $0.85 \pm 0.16$ & 0.671 & 0.512 \\
\hline & & AST & $23.87 \pm 12.93$ & $24.83 \pm 23.61$ & $22.50 \pm 6.07$ & 0.271 & 0.763 \\
\hline & & ALT & $28.79 \pm 22.76$ & $28.95 \pm 24.86$ & $19.88 \pm 8.87$ & 1.761 & 0.173 \\
\hline
\end{tabular}

${ }^{\star} P<0.05,{ }^{\star \star} P<0.01$.

disease decreased to $63 \%$ (OR: 0.37, 95\% CI: $0.21 \sim 0.65$ ) in the group that participated in high-intensity physical activity for 60 minutes or more per week compared to the group that participated for less than 10 minutes per week. The incidence of metabolic disease still decreased to 64\% (OR : 0.36, 95\% CI : 0.19 0.70) when the study was controlled for the age, body mass index, blood pressure, insulin, fasting glucose level and family history of diabetes.
It has been reported that physical activity contributes to decreased levels of TC and TG, which are related to various cardiovascular and metabolic diseases, including arteriosclerosis, as well as to an increase in HDL-C, which helps prevent diseases [29]. In this study, high-intensity physical activity was found to induce statistically significant differences in HDL-C in both the normal group $(P<0.01)$ and the metabolic disease group $(P<0.05)$ of $30 \sim 49$ year 
Table 6. Physical, hematological, and biochemical characteristics according to physical activity by age ( $50 \sim 69$ years)

\begin{tabular}{|c|c|c|c|c|c|c|c|}
\hline \multicolumn{3}{|c|}{ Characteristic } & \multirow{2}{*}{$\frac{\text { Low }}{16}$} & \multirow{2}{*}{$\frac{\text { Moderate }}{5}$} & \multirow{2}{*}{$\begin{array}{c}\text { High } \\
-\end{array}$} & \multirow[t]{2}{*}{$\mathrm{F}$} & \multirow[t]{2}{*}{$P$} \\
\hline Physical & Normal & $\mathrm{N}$ & & & & & \\
\hline & & Age & $57.31 \pm 5.81$ & $52.60 \pm 3.72$ & - & 2.867 & 0.107 \\
\hline & & WC & $77.59 \pm 7.03$ & $86.16 \pm 13.05$ & - & 3.741 & 0.068 \\
\hline & & BMI & $22.64 \pm 2.52$ & $24.72 \pm 2.81$ & - & 2.466 & 0.133 \\
\hline & & SBP & $104.13 \pm 9.10$ & $103.40 \pm 2.51$ & - & 0.030 & 0.864 \\
\hline & & DBP & $69.44 \pm 6.66$ & $69.40 \pm 3.91$ & - & 0.000 & 0.991 \\
\hline & Metabolic & N & 723 & 177 & 42 & & \\
\hline & & Age & $59.59 \pm 5.56$ & $59.15 \pm 5.43$ & $59.79 \pm 5.61$ & 0.492 & 0.612 \\
\hline & & WC & $85.16 \pm 8.93$ & $84.60 \pm 8.22$ & $84.54 \pm 8.60$ & 0.356 & 0.701 \\
\hline & & BMI & $24.92 \pm 3.20$ & $24.81 \pm 2.73$ & $24.21 \pm 2.97$ & 1.087 & 0.338 \\
\hline & & SBP & $126.21 \pm 17.29$ & $123.11 \pm 15.32$ & $128.02 \pm 15.66$ & 2.825 & 0.060 \\
\hline & & DBP & $78.18 \pm 10.39$ & $78.36 \pm 10.00$ & $80.24 \pm 10.55$ & 0.790 & 0.454 \\
\hline \multirow[t]{12}{*}{ Hematological } & Normal & $\mathrm{N}$ & 16 & 5 & - & & \\
\hline & & WBC & $6.16 \pm 1.05$ & $5.32 \pm 1.11$ & - & 2.369 & 0.140 \\
\hline & & $\mathrm{RBC}$ & $4.35 \pm 0.28$ & $4.47 \pm 0.49$ & - & 0.528 & 0.476 \\
\hline & & $\mathrm{HB}$ & $13.91 \pm 0.96$ & $14.36 \pm 1.58$ & - & 0.626 & 0.439 \\
\hline & & $\mathrm{HCT}$ & $41.02 \pm 2.69$ & $41.98 \pm 3.96$ & - & 0.391 & 0.539 \\
\hline & & PLT & $250.88 \pm 41.46$ & $269.60 \pm 48.65$ & - & 0.720 & 0.407 \\
\hline & Metabolic & $\mathrm{N}$ & 723 & 177 & 42 & & \\
\hline & & WBC & $6.42 \pm 1.75$ & $6.19 \pm 1.83$ & $6.50 \pm 2.12$ & 1.351 & 0.259 \\
\hline & & RBC & $4.59 \pm 0.41$ & $4.60 \pm 0.41$ & $4.74 \pm 0.42$ & 2.597 & 0.075 \\
\hline & & $\mathrm{HB}$ & $14.31 \pm 1.45$ & $14.46 \pm 1.28$ & $14.93 \pm 1.30$ & $4.440^{\star}$ & 0.012 \\
\hline & & $\mathrm{HCT}$ & $42.27 \pm 3.74$ & $42.55 \pm 3.59$ & $44.01 \pm 3.40$ & $4.559^{\star}$ & 0.011 \\
\hline & & PLT & $251.00 \pm 57.40$ & $244.97 \pm 54.19$ & $243.45 \pm 66.32$ & 1.037 & 0.355 \\
\hline \multirow[t]{18}{*}{ Biochemical } & Normal & $\mathrm{N}$ & 16 & 5 & & & \\
\hline & & FBS & $88.63 \pm 5.95$ & $89.00 \pm 5.79$ & - & 0.015 & 0.903 \\
\hline & & TC & $167.69 \pm 21.20$ & $173.40 \pm 10.55$ & - & 0.329 & 0.573 \\
\hline & & TG & $74.81 \pm 21.26$ & $73.20 \pm 14.18$ & - & 0.025 & 0.876 \\
\hline & & $\mathrm{HDL}$ & $53.75 \pm 10.70$ & $51.36 \pm 9.07$ & - & 0.203 & 0.658 \\
\hline & & BUN & $14.25 \pm 3.40$ & $13.00 \pm 2.65$ & - & 0.563 & 0.462 \\
\hline & & $C R$ & $0.70 \pm 0.13$ & $0.77 \pm 0.12$ & - & 1.069 & 0.314 \\
\hline & & AST & $20.63 \pm 3.52$ & $22.80 \pm 4.15$ & - & 1.345 & 0.260 \\
\hline & & ALT & $17.06 \pm 6.81$ & $17.40 \pm 5.03$ & - & 0.010 & 0.920 \\
\hline & Metabolic & N & 723 & 177 & 42 & & \\
\hline & & FBS & $117.50 \pm 30.10$ & $116.25 \pm 26.41$ & $114.17 \pm 17.10$ & 0.359 & 0.698 \\
\hline & & TC & $200.93 \pm 40.99$ & $200.08 \pm 38.15$ & $191.02 \pm 38.03$ & 1.201 & 0.301 \\
\hline & & $\mathrm{TG}$ & $171.88 \pm 117.50$ & $155.44 \pm 89.54$ & $152.26 \pm 98.78$ & 1.962 & 0.141 \\
\hline & & $\mathrm{HDL}$ & $48.98 \pm 11.87$ & $49.92 \pm 10.33$ & $51.78 \pm 14.80$ & 1.424 & 0.241 \\
\hline & & BUN & $15.79 \pm 4.58$ & $15.61 \pm 3.90$ & $16.02 \pm 5.57$ & 0.185 & 0.831 \\
\hline & & $C R$ & $0.84 \pm 0.25$ & $0.84 \pm 0.19$ & $1.04 \pm 0.80$ & $9.472^{* \star}$ & 0.000 \\
\hline & & AST & $25.44 \pm 16.63$ & $24.66 \pm 11.62$ & $26.43 \pm 13.20$ & 0.281 & 0.755 \\
\hline & & ALT & $25.81 \pm 19.86$ & $24.27 \pm 13.48$ & $23.52 \pm 14.18$ & 0.712 & 0.491 \\
\hline
\end{tabular}

${ }^{\star} p<0.05,{ }^{*} p<0.01$.

olds. These results are consistent with those reported [30-32]. Overall, these findings show that high-intensity physical activity increases HDL-C and is effective at preventing coronary artery diseases and metabolic diseases among 30 49 year olds. In addition, several previous studies have reported that high-intensity physical activity affects the composition and function of erythrocytes that constitute blood [33]. In the present study, we also found an increase in erythrocyte-related parameters in the normal group of 10 29 year olds and the metabolic disease group of 50 69 year olds when high-intensity physical activity was performed. The effects of regular physical activity vary depending on the intensity of physical activity. Participation in highintensity physical activity has a greater effect on reducing the risk of developing high cholesterol, diabetes, and 
152 Hyo Kyung Lim, et al. Relationship of Metabolic Diseases with Physical Activity Depending on Age

Table 7. Effects of age and physical activity intensity on $\mathrm{HDL}-\mathrm{C}$ in the normal and metabolic disease groups

\begin{tabular}{|c|c|c|c|c|c|c|c|c|}
\hline \multirow{2}{*}{ Group } & \multirow{2}{*}{$\begin{array}{l}\text { Independent } \\
\text { variable }\end{array}$} & \multicolumn{2}{|c|}{$\begin{array}{l}\text { Non-standardization } \\
\text { factor }\end{array}$} & \multirow{2}{*}{$\begin{array}{c}\begin{array}{c}\text { Standardization } \\
\text { factor }\end{array} \\
\beta\end{array}$} & \multirow[t]{2}{*}{$\mathrm{t}$} & \multicolumn{2}{|c|}{ Collinearity statistic } & \multirow[t]{2}{*}{$\mathrm{F}$} \\
\hline & & B & Se & & & tolerance & VIF & \\
\hline \multirow[t]{4}{*}{ Normal } & Constant & 53.42 & 1.98 & \multicolumn{3}{|c|}{$27.015^{\star \star}$} & & \multirow[t]{4}{*}{1.888} \\
\hline & Age & 0.05 & 0.04 & 0.05 & 1.010 & 0.978 & 1.022 & \\
\hline & Activity & 1.51 & 0.84 & 0.09 & 1.791 & 0.978 & 1.022 & \\
\hline & & & $R^{2}=0$ & , adjusted $R^{2}=0$. & $05, D W=1$. & & & \\
\hline \multirow[t]{4}{*}{ Metabolic } & Constant & 51.76 & 1.48 & & $35.077^{\star \star}$ & & & \multirow{4}{*}{$11.010^{\star \star}$} \\
\hline & Age & -0.08 & 0.02 & -0.09 & $-3.462^{\star \star}$ & .990 & 1.011 & \\
\hline & Activity & 1.53 & 0.55 & 0.07 & $2.798^{\star}$ & .990 & 1.011 & \\
\hline & \multicolumn{7}{|c|}{$R^{2}=0.015$, adjusted $R^{2}=0.013, D W=1.905$} & \\
\hline
\end{tabular}

${ }^{\star} P<0.05,{ }^{*} p<0.01$.

Abbreviation: VIF, Variance inflation factor.

hypertension, which are associated with metabolic diseases, than low-intensity or moderate-intensity physical activity. In a study of 49,005 adults, Williams and Thompson showed that the group participating in running, which is a high-intensity physical activity, had a lower risk of developing various diseases than the group participating in walking, which is a moderate-intensity physical activity [34]. Specifically, the risk of developing hypertension was 38\% (Hazard Ratios (HR): 0.62, 95\% CI: $0.55 \sim 0.70$ ) and the risk of developing diabetes was $71 \%$ (HR: 0.29, 95\% CI: 0.21 0.40). Regular physical activity has been shown to improve health, prevent chronic illnesses and have positive effects on mental health. As a result, the World Health Organization (WHO) and many countries, including the United States, Canada, Australia and Japan, have announced physical activity recommendations. It should be noted that the present study has the following limitations. The physical activity intensity of walking varies according to the speed of walking. For example, it can be 2.5 METs when you walk at $3.2 \mathrm{kph}$ and 8.0 METs when you walk at $8 \mathrm{kph}$. Moreover, it is affected by diverse factors, including weight of the load, carrying a baby, and geographical factors such as slope [35]. Therefore, it is not desirable to calculate the physical activity intensity of walking on the basis of METs of a single person. To enable more accurate measurement of the total physical activity, the validity of the method of calculating intensity of moderate-intensity physical activity, that is, the validity of calculating physical activity intensity in terms of average METs, needs to be discussed in the future research. Deriving the MET-min or kcal by calculating the intensity in terms of the average METs does not reflect the fact that a level of physical activity intensity may actually refer to a range of considerably different levels of intensity. While there was no difference in the levels of HDL-C observed according to age and physical activity intensity in the normal group, the level of HDL-C decreased with increasing age and increased with exercise intensity in the metabolic disease group. Physical activity has a different effect depending on age in the metabolic disease.

\section{요 약}

본 연구의 목적은 2014년 조사되어진 제 6기 2차 국민건강영 양조사자료를 바탕으로 연령별 신체활동에 따른 대사성질환과 의 관계를 파악하여 연령별 신체활동에 따른 대사성질환과의 관련성을 규명하여 예방적 기초자료를 제공하기 위한 연구이 다. 본 연구는 제 6기 2차(2014) 국민건강영양조사의 자료를 이 용하여 수행되었다. 정상군의 382 명과 대사질환군의 1,525 명 을 총 9,701 명의 설문 응답자 중 관련 자료가 없는 2,506 명을 제 외하고 총 7,295명을 분석했다. 본 연구에서 신체활동은 국제 신체활동 설문지 (IPAQ)를 기반으로 재분류 되었다. 대사증후 군의 정의는 2004년 개정 된 NCEP-ATP III에 근거하여 다음과 같은 결론을 얻었다. 연령에 따른 대사성질환과 신체활동과의 관계에서 10 29세 정상군과 50 69세의 대사질환군에서 혈 색소, 적혈구용적, 크리아티닌의 수치는 고강도 신체활동이 수 행되었을 때 증가했고 저강도 신체활동을 수행되었을 때 감소 했다. 30 49세의 정상군과 대사질환군에서 고밀도 지단백 콜 
레스테롤 수치는 고강도 신체활동이 수행되었을 때 증가했지 만, 저강도 신체활동이 수행되었을 때 감소하였다. 따라서 연령 과 운동강도가 고밀도 지단백 콜레스테롤 수치에 미치는 영향 을 조사하였다. 결과는 정상군에서 연령과 운동강도에 따라 고 밀도 지단백 콜레스테롤 수치에 차이는 없었지만 대사질환군에 서는 연령에 따라 고밀도 지단백 콜레스테롤 수치가 감소하였 고 운동강도에 따라 증가하였다. 종합하면, 본 연구의 결과는 대 사질환군에서 고밀도 지단백 콜레스테롤 수치는 고강도 신체활 동에서 긍정적인 효과를 나타내고 연령은 부정적인 효과를 나 타냈다. 이러한 결과는 우리가 신체활동과 연령에 따라 대사성 질환을 더 잘 이해하는데 도움이 될 수 있다.

\section{Acknowledgements: None \\ Conflict of interest: None}

\section{REFERENCES}

1. Ford ES. Risks for all-cause mortality, cardiovascular disease, and diabetes associated with the metabolic syndrome: a summary of the evidence. Diabetes Care. 2005;28:1769-1778.

2. Coan PM, Barrier M, Alfazema N, Carter RN, Marion de Procé S, Dopico XC, et al. Complement factor B is a determinant of both metabolic and cardiovascular features of metabolic syndrome. Hypertension. 2017;70:624-633.

3. Primeau V, Coderre L, Karelis AD, Brochu M, Lavoie ME, Messier V, et al. Characterizing the profile of obese patients who are metabolically healthy. Int J Obes. 2011;35:971-981.

4. Grundy SM, Cleeman JI, Daniels SR, Donato KA, Eckel RH, Franklin BA, et al. Diagnosis and management of the metabolic syndrome: an American Heart Association/National Heart, Lung, and Blood Institute Scientific Statement. Circulation. 2005;112:2735-2752.

5. Ford ES, Giles WH, Dietz WH. Prevalence of the metabolic syndrome among US adults: findings form the third National Health and Nutrition Examination Survey. JAMA. 2002;287: 356-359.

6. Oh SW. Obesity and metabolic syndrome in Korea. Diabetes Metab J. 2001;35:561-566.

7. Kelly RB, Zyzanski SJ, Alemagno SA. Prediction of motivation and behavior change following health promotion: role of health beliefs, social support, and self-efficacy. Soc Sci Med. 1991;32:311-320.

8. Laaksonen, DE, Lakka HM, Salonen JT, Niskanen LK, Rauramaa R, Lakka TA. Low levels of leisure time physical activity and cardiorespiratory fitness predict development of the metabolic syndrome. Diabetes Care. 2002;25:1612-1618.

9. Lakka TA, Laaksonen DE, Lakka HM, Mannikko N, Niskanen LK, Rauramaa R, et al. Sedentary lifestyle, poor cardiorespiratory fitness, and the metabolic syndrome. Med Sci Sports Exerc. 2003;35:1279-1286.

10. Chrysohoou C, Skoumas J, Georgiopoulos G, Liontou C,
Vogiatzi G, Tsioufis K, et al. Exercise capacity and haemodynamic response among 12,327 individuals with cardio-metabolic risk factors undergoing treadmill exercise. Eur J Prev Cardiol. 2017;24:1627-1636.

11. Levine GN, Baldady GJ. The benefits and risks of exercise traning: the exercise prescription. Adv Intern Med. 1993;38:57-79.

12. McMurdo, ME, Lennie L. A controlled trial of exercise by residents of old people's homes. Age Ageing. 1993;22:11-15.

13. Limacher MC. Aging and cardiac function: influence of exercise. South Med J. 1994;87( Suppl 5):13-16.

14. Greig CA, Young A, Skelton DA, Pippet E, Butler FM, Mahmud SM. Exercise studies with elderly volunteers. Age Ageing. 1994;23:185-189.

15. Centers for Disease Control and Prevention. Physical activity and health: a report of the surgeon general. Atlanta, US Department of Health and Human Services; 1996.

16. Oja P, Vuori I, Paronen O. Daily walking and cycling to work: their utility as health-enhancing physical activity. Patient Educ Couns. 1998;33(Suppl 1):87-94.

17. Helgerud J, Hoydal K, Wang E. Aerobic high-intensity intervals imprrove VO2max more then moderate training. Med Sci Sports Exerc. 2007;39: 665-671.

18. Heyward VH, Gibson AL. Advanced fitness assessment and exercise prescription. 7th ed. Champaign: Human kinetics; 2014.

19. Swain DP, Franklin BA. Comparison of cardioprotective benefits of vigorous versus moderate intensity aerobic exercise. Am J Cardiol. 2006;97:141-147.

20. Rennie KL, McCarthy N, Yazdgerdi S, Marmot M, Brunner E. Association of the metabolic syndrome with both vigorous and moderate physical activity. Int J Epidemiol. 2003;32:600-606.

21. Torok, K, Szelenyi Z, Porszasz J, Molnar D. Low physical performance in obese adolescent boys with metabolic syndrome. Int J Obes Relat Metab Disord. 2001;25:966-970.

22. Jurca R, Lamonte MJ, Church TS, Earnest CP, Fitzgerald SJ, Barlow CE, et al. Associations of muscle strength and fitness with metabolic syndrome in men. Med Sci Sports. 2004;36: 1301-1307.

23. Ministry of Health and Welfare, Korea Centers for Disease Control and Prevention. Korea Health Statistics 2014: Korea National Health and Nutrition Examination Survey (KNHANES VI-2). Report. Cheongju: Korea Centers for Disease Control and Prevention; 2015.

24. Expert Panel on Detection, Evaluation, and Treatment of High Blood Cholesterol in Adults. Executive summary of the third report of the national cholesterol education program (NCEP) expert panel on detection, evaluation, and treatment of high blood cholesterol in adults (Adult Treatment Panel III). JAMA. 2001;285:2486-2497.

25. Chun NY. Validity and reliability of Korean version of international physical activity questionnaire short form in the elderly. Kor J Fam Med. 2012;33:144-151.

26. International Consensus Group for the Development of an International Physical Activity Questionnaire. Guidelines for data processing and analysis of the International Physical Activity Questionnaire [Internet]. Geneva: WHO; 2005 [2018 May 23]. Available from: file://C:/Users/Administrator/ Downloads/GuidelinesforDataProcessingandAnalysisoftheInter 
nationalPhysicalActivityQuestionnaireIPAQShortandLongForm s.pdf.

27. Tjonna AE, Lee SJ, Rognmo O, Stolen TO, Bye A, Haram PM, et al. Aerobic interval training versus continuous moderate exercise as a treatment for the metabolic syndrome a pilot study. Circulation. 2008;118:346-354.

28. Srivastava RAK. Dysfunctional HDL in diabetes mellitus and its role in the pathogenesis of cardiovascular disease. Mol Cell Biochem. 2018;440:167-187.

29. Goldberg L, Elliot DL, Schutz RW, Kloster FE. Changes in lipid and lipoprotein levels after weight training. JAMA. 1984;252: 504-506.

30. Sady SP, Berg K, Beal D, Smith JL, Savage MP, Thompson WH, et al. Aerobic fitness and serum HDL-C in young children. Hum Biol. 1984;56:771-782.
31. Spoko G, Obarzanek E, Stone E. Overview of the national healt, lung, and blood institute workshop on physical activity and cardiovascular health. Med Sci Sports Exer. 1992;24:192-195.

32. Li N, He S, Blomback M, Hjemdahl P. Platelet activity coagulation and fibrinolysis during exercise in health males; Effects of thrombin inhibition by argatroban and enoxaparin. Arterioscle Thromb Vasc Biol. 2007;27:407-413.

33. Williams PT, Thompson PD. Walking versus running for hypertension, cholesterol, and diabetes mellitus risk reduction. Arterioscler Thromb Vasc Biol. 2013;33:1085-1091.

34. Ainsworth B, Haskell WL, White MC, Irwin ML, Swartz AM, Strath SJ, et al. Compendium of physical activities: an update of activity codes and MET intensities. Med Sci Sports Exerc. 2000; 32(9 Suppl):498-516. 